\title{
Effect and mechanism of different ways of transplanting bone marrow mesenchymal stem cells in cardiopulmonary resuscitation in rats
}

\author{
L.L. Zhou ${ }^{1,2}$, J.K. Liang ${ }^{1,2}$, Q.M. Lin ${ }^{1,2}$ and Z.T. Huang ${ }^{1,2}$ \\ ${ }^{1}$ Sun Yat-Sen Memorial Hospital, Sun Yat-Sen University, Guangzhou, China \\ ${ }^{2}$ Institute of Cardiopulmonary Cerebral Resuscitation, Sun Yat-Sen University, \\ Guangzhou, China
}

Corresponding author: Z.-T. Huang

E-mail: syxhzt@sina.cn / zhoulili_1980@163.com

Genet. Mol. Res. 13 (3): 7937-7949 (2014)

Received August 12, 2013

Accepted December 5, 2013

Published September 29, 2014

DOI http://dx.doi.org/10.4238/2014.September.29.7

\begin{abstract}
The effect and mechanism of different ways of transplanting bone marrow mesenchymal stem cells (MSCs) were explored for treating cardiopulmonary resuscitation in a rat model. Rats were divided into the cardiopulmonary resuscitation group (Group Con), the stereotactic lateral ventricle transplantation group (Group LV), the internal carotid artery transplantation group (Group A), and the femoral vein transplantation group (Group V). MSCs were transplanted in Groups LV, A, and V. The MSC transplantation groups had a significantly higher neurological score than Group Con on days 3 and 7 after recovery $(\mathrm{P}<0.05)$, and the Group LV score was the highest in all groups $(\mathrm{P}<0.05)$. On day 3 after recovery, the MSC count of Group LV was significantly higher than those of Groups A and V $(\mathrm{P}<0.01)$. On day 7 after recovery, the MSC count in the hippocampus of Group LV was significantly higher than those of Groups $\mathrm{A}$ and $\mathrm{V}(\mathrm{P}<0.05)$. On day 3 after recovery, the S100B level of Group LV was significantly lower than those of Groups A and V $(\mathrm{P}<0.05)$.
\end{abstract}


On day 7 after recovery, the S100B level was not significantly different between the MSC transplantation groups $(\mathrm{P}>0.05)$. The expressions of vascular endothelial growth factor and brain-derived neurotrophic factor did increased significantly in the MSC transplantation groups on day 7 after recovery, particularly Group LV. Therefore, MSC therapy could significantly improve nerve function after cardiopulmonary resuscitation, and stereotactic lateral ventricle injection transplantation is an optimal method.

Key words: Bone marrow mesenchymal stem cell; Neurological score; Cardiopulmonary resuscitation

\section{INTRODUCTION}

Bone marrow mesenchymal stem cells (BMSCs) are a type of stem cell with the potential for proliferation and multi-directional differentiation. BMSCs can differentiate into neural cells both in vivo and ex vivo (Shi and Yang, 2012). In recent years, BMSC transplantation showed important progress in the treatment of stroke (Wei et al., 2012; He et al., 2013), myocardial infarction, and nervous system recovery after cardiopulmonary resuscitation (Wen et al., 2012; Williams et al., 2013). Therefore, BMSC transplantation has become a new technique for treating diseases of the nervous system. At present, most of the applied researchs on repairing nerve tissues by BMSCs have focused on the focal cerebral ischemia model. For whole brain ischemic injury, MSC transplantation can reduce brain damage as well as improve nerve function (Wang et al., 2011).

Cardiac arrest is the most important cause of whole cerebral hypoxic-ischemic damage. The most vulnerable areas are the hippocampus, cortex, thalamus, and cerebellum, wherein whole cerebral hypoxic-ischemia can lead to necrosis and apoptosis of a large number of neuronal cells in a short time, causing nerve dysfunction. How to reduce and even prevent such neuronal damage, and to promote neural functional recovery after cardiac arrest has become an important issue in the field of cardiopulmonary resuscitation research.

This study considered three different ways of transplanting 4-6-diamidino-2-phenylindole (DAPI)-labeled MSCs in rats after cardiopulmonary resuscitation: stereotactic lateral ventricle infusion, internal carotid artery infusion, and femoral vein infusion. The effects of different transplantation methods on cerebral resuscitation after cardiac arrest in rats were then observed.

\section{MATERIAL AND METHODS}

\section{Experimental animals}

Healthy Sprague-Dawley (SD) rats, 4-5 weeks old, weighing 100-150 g, and healthy SD rats weighting 300-400 g were provided by the Experimental Animal Center of Sun YatSen University.

\section{Isolation, culture, subculture, and identification of MSCs}

SD rats aged 4-5 weeks were executed using the cervical dislocation method, and 
were disinfected by immersion in $75 \%$ alcohol for $5 \mathrm{~min}$. The skin was removed and the rats were dissected under sterile conditions, removing the muscles and soft tissue, picking out both sides of the femur and tibia, and placing them on a Petri dish on clean benches. The double ends of the femur and the tibia were cut open, and the bone cavity was repeatedly rinsed with Dulbecco's modified Eagle's medium/Ham's F12 (DMEM/F12) without fetal bovine serum (FBS) until the cavity became white. The centrifugal tube was filled with lavage fluid, and the supernatant was removed after centrifuging at $1500 \mathrm{rpm}$ for $5 \mathrm{~min}$. The sediment was placed into $8 \mathrm{~mL}$ DMEM per rat. A 4-mL mixture was extracted, placed in $25-\mathrm{cm}^{2}$ culture flasks, and cultured in a tri-gas incubator. Half of the medium was changed after $24 \mathrm{~h}$, the whole medium was changed after $48 \mathrm{~h}$, and the medium was changed every other day thereafter.

The DMEM was sucked out when $90 \%$ of the cells fused (6-7 days). The bottom of the bottle was rinsed with phosphate-buffered saline (PBS), and $3 \mathrm{~mL} 0.25 \%$ pancreatin containing $0.1 \mathrm{mM} 0.25 \%$ ethylenediaminetetraacetic acid (EDTA) was added to the culture flask for digestion for $2 \mathrm{~min}$ at $37^{\circ} \mathrm{C}$.

Digestion in the culture flask was observed using an inverted microscope. Digestion was stopped by adding DMEM when the flask was filled with MSCs and a portion of them floated. The DMEM was imbibed using a pipette, and the liquid was transferred to a centrifuge tube. The supernatant was removed after centrifuging at $1500 \mathrm{rpm}$ for $5 \mathrm{~min}$. The cell pellets were resuspended in DMEM, inoculated, and passaged (1:2), and the above amplification subculture procedures were repeated when $90 \%$ of the cells fused (about 2-3 days).

Cells of the $\mathrm{P} 3$ generation were digested with $0.25 \%$ pancreatin at $90 \%$ cell fusion, and were washed three times with PBS. Fluorescence-labeled CD44, CD34, CD45, and CD90 antibodies were respectively added and incubated for $30 \mathrm{~min}$ at $4^{\circ} \mathrm{C}$. Unlabeled antibody was fixed with $1 \%$ paraformaldehyde and washed with PBS after 15 min. Flow cytometry was used to detect the expression of the surface antigens of the MSCs.

The MSCs were collected at the P3 generation of growth, the DMEM was sucked out, and the cells were washed three times with PBS. Four milliliters of DMEM was added and marked with $50 \mu \mathrm{g} / \mathrm{mL}$ DAPI nucleus-staining solution in the culture flask. The culture was incubated for $2 \mathrm{~h}$ at $37^{\circ} \mathrm{C}$, with $5 \% \mathrm{CO}_{2}$ saturated humidity for cell transplantation.

\section{Rat model of cardiac arrest resuscitation}

SD rats were randomly divided into different groups before establishing the models: the cardiopulmonary resuscitation group (Group Con), the stereotactic lateral ventricle transplantation group (Group LV), the internal carotid artery transplantation group (Group A), and the femoral vein transplantation group (Group V). Each group was divided into subgroups according to 3 and 7 days after restoration of spontaneous circulation (ROSC) (3-day and 7-day subgroups). The cardiac arrest resuscitation model was established using the smothering method. Group Con received cardiopulmonary resuscitation only without MSC transplantation $(\mathrm{N}=8)$.

Group LV received MSC transplantation to the lateral ventricle using a stereotaxis instrument after ROSC $(\mathrm{N}=8)$; Group A received MSC transplantation via the unilateral internal carotid artery after ROSC $(\mathrm{N}=8)$; Group V received MSC transplantation via the unilateral femoral vein after ROSC $(\mathrm{N}=8)$.

The transplantation of MSCs occurred $1 \mathrm{~h}$ after ROSC in all groups, with approximately $1 \times 10^{6}$ cells transplanted. 


\section{Model establishment}

Healthy male SD rats weighing between 300 and $400 \mathrm{~g}$ were selected and fasted for 24 $\mathrm{h}$ before modeling, except water. After induction of anesthesia with $\mathrm{CO}_{2}, 45 \mathrm{~g} / \mathrm{L} 0.1 \mathrm{~mL} / 100$ g sodium pentobarbital solution was intraperitoneally injected. After a successful anesthesia effect, the skin of the chest, back, and groin was prepared. The rats were placed in supine position on the operating table. Endotracheal intubation was performed under direct vision using head mirror reflection and a focused light source. A tracheal tube was fixed to the lower lip with suture after successful intubation, and the carbon dioxide monitor was linked to track the $\mathrm{ETCO}_{2}$ concentration. One side of the lower extremity skin was cut after disinfection with a blunt dissection of the femoral artery while blocking the blood flow with ophthalmic forceps. The distal end of the femoral artery was ligated, a break was cut above the ligation point using ophthalmic scissors, a 23-PE heparinized pipe was placed, and the tube was inserted to the thoracic aorta along the femoral artery (about $3-4 \mathrm{~cm}$ ). The catheter was fixed with suture and connected to the pressure sensor of a multi-channel physiological parameter instrument. The mean arterial pressure, body temperature, and electrocardiogram changes of experimental rats were continuously monitored.

After completing the surgical procedure, the baseline physiological parameters were recorded when rats were fully awake. One milligram of vecuronium per kilogram was injected via a tee connected to the catheter, until breathing gradually stopped after $10 \mathrm{~s}$. Changes in blood pressure were observed after the complete cessation of breathing. The standard criteria of cardiac arrest are an arterial blood pressure without fluctuation and a mean arterial pressure falling to $20 \mathrm{mmHg}$ (about 4-5 min). The countdown began upon initiating chest compressions using a cardiopulmonary resuscitation device after $6 \mathrm{~min}$. The compression rate was $200 \mathrm{bpm}$, the compression/ventilation ratio was $2: 1$, and the compression depth was $1 / 3$ of the anteroposterior diameter of the thorax. The adrenal was injected with $0.1 \mathrm{~mL}$ solution through the femoral artery after $2 \mathrm{~min}$, and washed with $0.1 \mathrm{~mL}$ heparin saline. The standard criteria of ROSC are a mean arterial pressure maintained above $60 \mathrm{mmHg}$ for more than 5 min under continuous chest compressions and $50 \%$ oxygen mechanical ventilation after giving pure oxygen ventilation for $15 \mathrm{~min}$. Recovery failure is considered if there is no ROSC after 5 min of chest compressions. ROSC mechanical ventilation was applied and hemodynamic changes were monitored.

\section{Transplantation of MSCs after recovery}

Upon close observation for 15 min after ROSC, the DAPI-labeled MSCs can be prepared for $2 \mathrm{~h}$ of transplantation as long as the systolic blood pressure is greater than $110 \mathrm{mmHg}$ and stability is maintained. DAPI-labeled MSCs were extracted randomly, washed five times with PBS, digested with $0.25 \%$ pancreatin, and terminated with FBS. The total number of cells was calculated using a cell counting board, the supernatant was removed after centrifuging at $1500 \mathrm{rpm}$ for $5 \mathrm{~min}$, and $0.5 \mathrm{~mL}$ PBS was added to resuspend the MSCs. The mixture fluid was placed into a 1-mL syringe. MSCs labeled with DAPI were injected into the rats after ROSC $1 \mathrm{~h}$ later via the different methods in each group.

\section{Stereotactic brain lateral ventricle transplantation procedure}

At the transplantation site, a lateral ventricle incision was made $0.72 \mathrm{~mm}$ after the 
anterior fontanelle, followed by the $1.6-\mathrm{mm}$ sagittal suture $3.2 \mathrm{~mm}$ under the subdural. For fixation, rats were placed in stereotactic shelves with heads fixed with incisors and ear holders. The incisor holders should be less than the level of the binaural line; $3.0 \pm 0.3 \mathrm{~mm}$. For skin preparation, head hair was removed using a razor to expose the skin near the anterior fontanelle. The surgical area was disinfected with povidone-iodine, and a $1.5-\mathrm{cm}$ midline incision was made. Blunt dissection of the periosteum was executed, exposing the anterior fontanelle, and sterile gauze was applied to stop the bleeding. The graft site was marked and located using a brain stereotaxic apparatus. Drilling was performed perpendicular to the cone drill into the skull.

For transplantation, approximately $15 \mu \mathrm{L}$ MSC mixture fluid was extracted, the stereotactic instrument was fixed, and a micro-syringe needle was inserted into a hole located at the graft site under the stereotactic apparatus with the bolus and slowly injected at $1 \mu \mathrm{L} / \mathrm{min}$. The quantity of cells desired was approximately $1 \times 10^{6}$; thus, the needle needed to stand for 20 min after bolus.

After the micro-syringe needle was removed from the hole, the area was covered with a sterile gelatin sponge, disinfected with sulfur crystalline powder, and the skin was sutured with the incision disinfected with povidone-iodine.

\section{Carotid artery transplantation procedure}

The unilateral neck skin was disinfected after successful resuscitation, the skin was cut in the outer part, and a blunt dissection of the carotid artery was performed, with the proximal end ligated and an incision cut out using ophthalmic scissors. A 23-PE-50 polyethylene pipe was heparinized and positioned toward the skull base at approximately $2 \mathrm{~cm}$ in depth. This was fixed with a suture, with the other end connected with tee fitting using a heparin saline-sealed tube. At $1 \mathrm{~h}$ after ROSC, a $0.5-\mathrm{mL}$ syringe equipped with MSCs connected the bolus slowly, and then saline was injected using a $0.2-\mathrm{mL}$ washpipe. The following procedures were performed: removal of the catheter, ligature of the distal end of the carotid artery, sutured incision, and povidone-iodine disinfection.

\section{Femoral vein transplantation procedure}

Blunt dissection of the femoral vein was performed at the same side after successful resuscitation. The distal end was ligated, and an incision was cut out using ophthalmic scissors. A 23-PE-50 polyethylene heparinized pipe was placed toward the skull base at approximately $2 \mathrm{~cm}$ in depth with the suture fixed. The other end was connected with tee fitting, and a heparin saline-sealed tube was used. At $1 \mathrm{~h}$ after ROSC, a $0.5-\mathrm{mL}$ syringe equipped with MSCs connected the bolus slowly, and then saline was injected using a $0.2-\mathrm{mL}$ washpipe. The following procedures were performed: removal of the catheter, ligature of the proximal end of the femoral artery, suture of incision, and povidone-iodine disinfection.

\section{Post-operation procedures}

All catheters were disconnected after MSC transplantations, and $0.2 \mathrm{~mL} 250 \mathrm{~g} / \mathrm{L}$ cephalosporin VI was given via intramuscular injection. The rats were returned to the experimental animal cages. Rats were fed the first day after ROSC in individually housed cages and the room 
temperature was maintained at about $25^{\circ} \mathrm{C}$. Five percent glucose saline injection artificial feeding was given every $6 \mathrm{~h}$ after surgery. The rats were returned to their cages 3 days later.

\section{Neurological score in rats}

The neurological functions of rats at days 1, 3, and 7 after ROSC were scored using the 80 neural function defect score (Neurological Deficit Scale Scores; NDSS) as a standard, as shown in Table 1.

\begin{tabular}{ll} 
Table 1. Neurological Deficit Scale Scores. & \\
\hline 1. Overall behavior & 19 points \\
Conscious & Normal $10 /$ stupor $5 /$ coma 0 \\
Wakefulness & Spontaneous open $3 /$ pain in the open $1 /$ non-open 0 \\
Breathe & Normal $6 /$ insufficient or excessive ventilation $3 /$ disappear 0 \\
2. Brain stem function & 21 points \\
Olfactory sensation (reaction to food and odors) & Exist $3 /$ disappear 0 \\
Vision (reaction to light) & Exist $3 /$ disappear 0 \\
Light reflex & Exist $3 /$ disappear 0 \\
Corneal reflex & Exist $3 /$ disappear 0 \\
Startle reflex & Exist $3 /$ disappear 0 \\
Whisker stimulus & Exist $3 /$ disappear 0 \\
Deglutition reflex & Exist $3 /$ disappear 0 \\
3. Campaign evaluation & 6 points \\
Myodynamia (evaluate each side) & Normal $3 /$ stiff or reduce $1 /$ no movement or paralysis 0 \\
4. Sensory assessment & 6 points \\
Pain (evaluate each side) & Sensitive to retreat $3 /$ buckling stretch $1 /$ no retreat 0 \\
5. Exercise behavior & 6 points \\
Gait coordination & Normal $3 /$ abnormal $1 /$ deficiency 0 \\
Beam balance & Normal $3 /$ abnormal $1 /$ deficiency 0 \\
6. Behavior & 12 points \\
Righting reflex & Normal $3 /$ abnormal $1 /$ deficiency 0 \\
Chemotaxis experiments & Normal $3 /$ abnormal $1 /$ deficiency 0 \\
Visual orientation & Normal $3 /$ abnormal $1 /$ deficiency 0 \\
Alley turned to experimental & Normal $3 /$ abnormal $1 /$ deficiency 0 \\
7. Seizures & 10 points \\
Convulsive or non-convulsive epilepsy & No tic $10 /$ focal convulsions / generalized convulsions 0 \\
\hline Remark: 80 points & and \\
\hline
\end{tabular}

Remark: 80 points $=$ normal; 0 point $=$ brain death. Beam balance test normal: rats can walk through beams $0.5 \mathrm{~m}$ high, $1 \mathrm{~m}$ long, $2 \mathrm{~cm}$ wide; abnormal: tried but failed to continue, or stopped for a moment then fell off; disappear: fell off as soon as rat got up.

\section{Tissue samples processing}

Forty-five grams per liter $0.1 \mathrm{~mL} / 100 \mathrm{~g}$ sodium pentobarbital solution was injected intraperitoneally after induction of anesthesia with $\mathrm{CO}_{2}$ upon reaching the observation point. With the rat fixed on the operating table, a blunt dissection of the common carotid artery and ligature of the proximal end of the common carotid artery were performed. An incision was cut out with ophthalmic scissors above the ligation point. A 23-PE-50 polyethylene heparinized pipe was positioned at the skull base with the other end of the tube connected to a syringe 
equipped with $4 \%$ paraformaldehyde; the chest was quickly cut open, and the heart was exposed. Paraformaldehyde was slowly injected from the internal carotid artery to the brain until the superior vena cava outflow was clear of paraformaldehyde; the process took no less than $20 \mathrm{~min}$. The rat head was quickly cut off after injection. The skull was opened, the left side of the brain was removed and placed in $4 \%$ paraformaldehyde, and soaked for 3 days for the production of paraffin-embedded slices.

\section{Detection of VEGF and BDNF contents by the immunohistochemical method}

Tissue sections were embedded with paraffin by SABC immunohistochemistry, lostwax hydration, and rinsed with double-distilled water and PBS. The antigen was maintained after $15 \mathrm{~min}$, then cooled to room temperature, and rinsed in PBS 3 times for 2 min each time. Deionized water was added at room temperature, incubated for $10 \mathrm{~min}$, and rinsed 3 times with PBS for 2 min each time. Each slice was incubated after drop-wise additions of anti-goat serum at room temperature. The tissue sections were stored at $4^{\circ} \mathrm{C}$ overnight and rinsed with PBS three times for 2 min each time. Biotinylated secondary antibody was incubated for $30 \mathrm{~min}$ at room temperature, and washed with PBS for 5 min 3 times. DAB was developed, and the reaction was controlled under a microscope. The slices were rinsed with distilled water, dyed with hematoxylin, differentiated using hydrochloric acid alcohol, and dehydrated and mounted with neutral gum.

\section{Method of immunoreactive scoring (IRS)}

The IRS was calculated as: IRS $=P \times I . P$ represents the percentage of positive cells: no positive cells, $P=0$; positive cells accounting for 1 to $24 \%, P=1$; positive cells accounting for 25 to $49 \%, P=2$; positive cells accounting for 50 to $74 \%, P=3$; positive cells accounting for 75 to $100 \%, P=4$. I represents the staining intensity: no staining, $\mathrm{I}=0$; mild staining, $\mathrm{I}=$ 1 ; moderate staining, $\mathrm{I}=2$; deep staining, $\mathrm{I}=3$. Five high-power fields were selected randomly from each specimen and were averaged.

\section{Data analysis}

The IBM SPSS Statistics 20.0 software for MAC was used to process and analyze the data. Measurement data are reported as means \pm standard deviations. Analysis of variance was performed for comparison between groups. All parametric data were analyzed using the chisquare statistic, and statistical significance was determined at $\mathrm{P}<0.05$.

\section{RESULTS}

\section{Neurological score in rats}

As shown in Figure 1, there were no significant differences in neurological score between the four groups on day 1 after recovery $(\mathrm{P}>0.05)$. However, the groups receiving MSC transplantation scored significantly higher than Group Con on days 3 and 7 after ROSC $(\mathrm{P}<$ $0.05)$ and the score of Group LV was higher than those of Groups A and V $(\mathrm{P}<0.05)$. There were no significant differences between Groups A and V $(\mathrm{P}>0.05)$. 


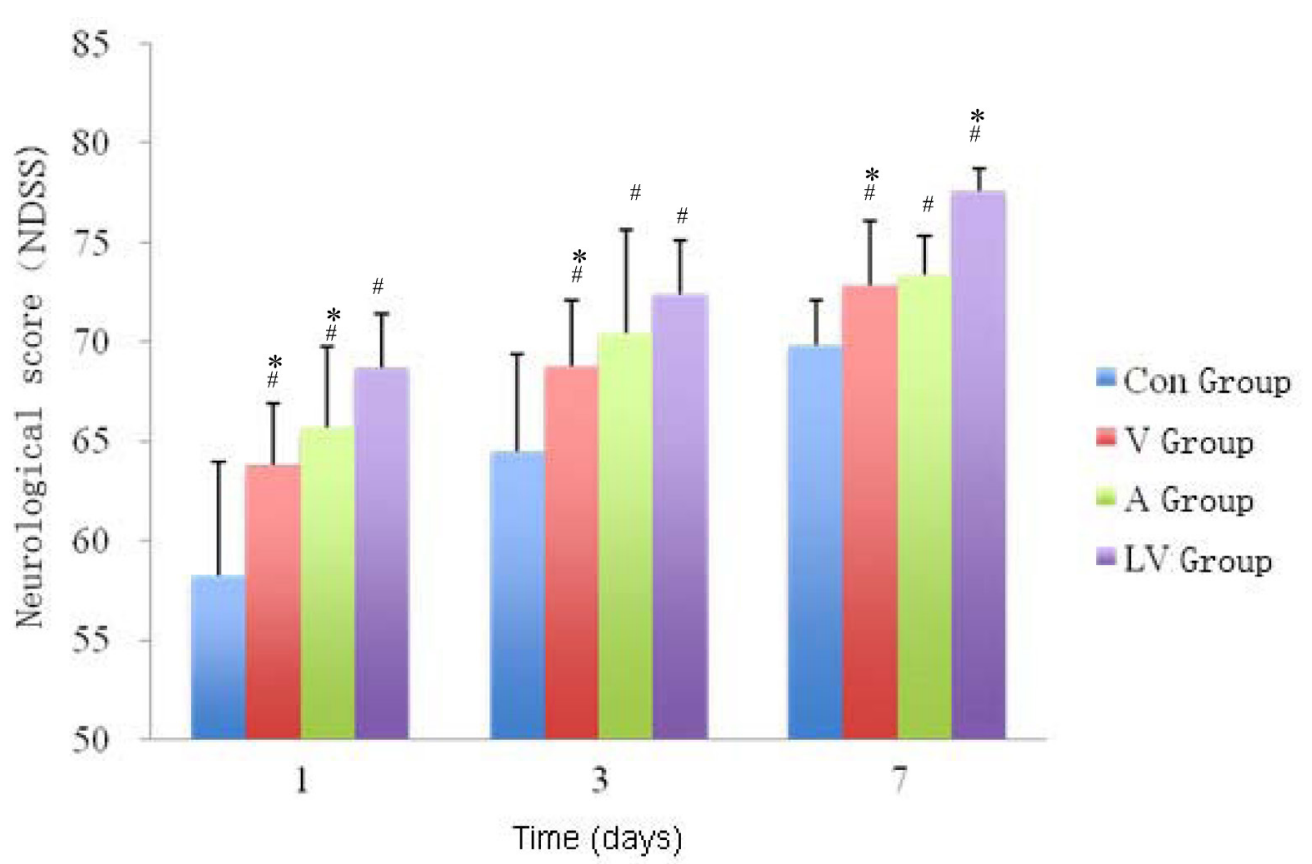

Figure 1. Neurological score in rats of different groups after restoration of spontaneous circulation. ${ }^{\#} \mathrm{P}<0.05$ vs Group Con; *P $<0.05$ vs Group LV. Con = control group; V, A, LV = intravenous, artery, stereotactic lateral ventricle groups, respectively.

\section{Distribution and migration of transplanted MSCs}

The transplanted DAPI-labeled MSCs were observed under a fluorescence microscope, and a $99 \%$ positive rate of DAPI staining was found. This indicated that DAPI-labeled MSCs can be used as a tracer in the body after transplantation.

We observed blue-marked cells in the brain tissue on day 3 after transplantation. MSCs aggregated in the area of the hippocampus and temporal cortex, which is consistent with the ischemia-vulnerable area of the global cerebral model. On day 3 after recovery, the MSC count of Group LV was significantly higher than those of Groups A and V $(\mathrm{P}<0.01)$; however, there were no significant differences between Groups A and V $(P>0.05)$. On day 7 after recovery, the MSC count in the hippocampus of Group LV was significantly higher than those of Groups A and V $(\mathrm{P}<0.05)$; however, the cell count in the temporal cortex area did not differ significantly between the four groups $(\mathrm{P}>0.05)$, as shown in Figures 2-4.

\section{Comparison of serum S100B levels}

As shown in Figure 5, the S100B level increased significantly in each group. The S100B serum levels of the MSC transplantation groups were significantly lower than that of Group Con $(\mathrm{P}<0.05)$ on days 3 and 7 after ROSC. On day 3 after ROSC, the S100B level of Group LV was significantly lower than those of Groups A and V $(\mathrm{P}<0.05)$; however, there were no significant differences between Groups A and V $(\mathrm{P}>0.05)$. On day 7 after ROSC, the 
S100B level gradually declined in all groups. There were no significant differences between Groups LV, A, and V $(\mathrm{P}>0.05)$.

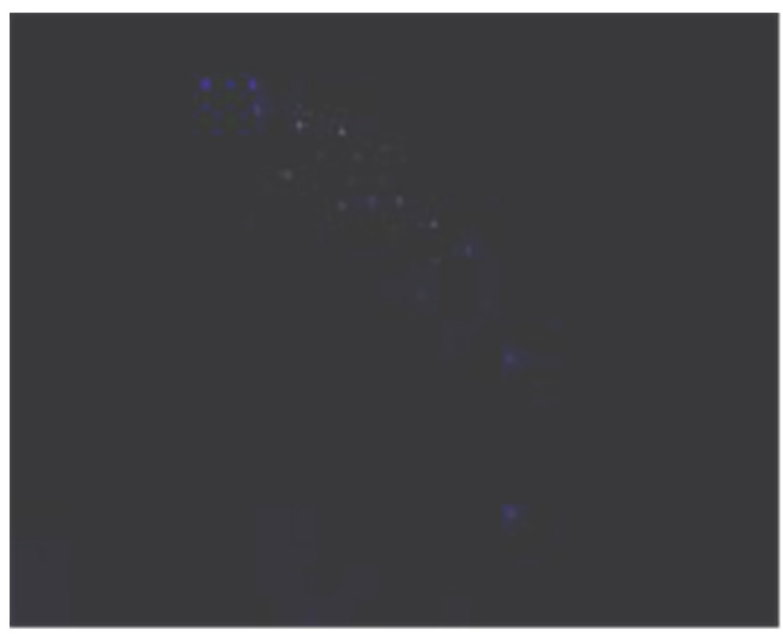

Figure 2. Cells labeled with DAPI in the hippocampus 3 days after MSC transplantation.

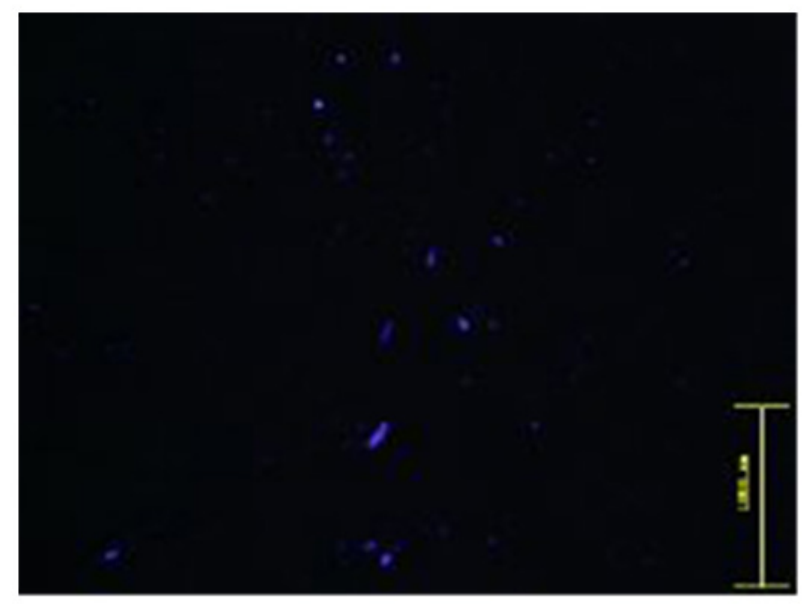

Figure 3. Cells labeled with DAPI in the temporal cortex 3 days after MSC transplantation.

\section{Observations of VEGF and BDNF in the brain tissue by immunohistochemistry}

The immunohistochemical staining results were in accordance with the IRS standard as shown in Figure 6. The expression levels of VEGF and BDNF did not change significantly in Group Con on day 7 after ROSC. There was a significant increase in VEGF and BDNF in the groups that received MSC transplantation. The expressions of VEGF and BDNF in Group LV were obviously higher than those of Groups A and V. There were no significant differences between Groups A and V, as shown in Figure 7. 


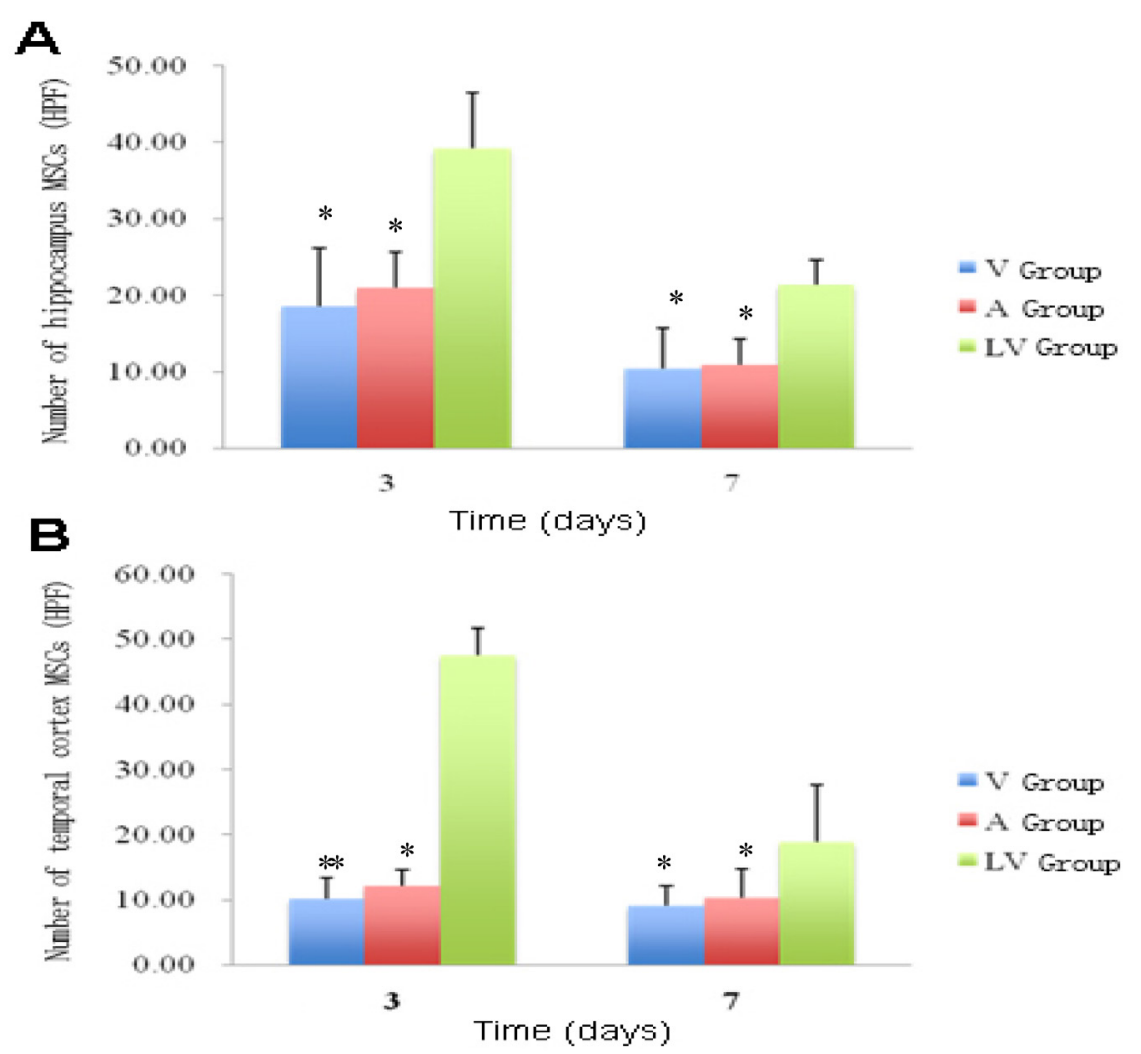

Figure 4. Distribution of MSCs in the hippocampus and temporal cortex of the transplantation groups. $* * \mathrm{P}<0.01$ vs Group LV; *P $<0.05$ vs Group LV. For group abbreviations, see legend to Figure 1.

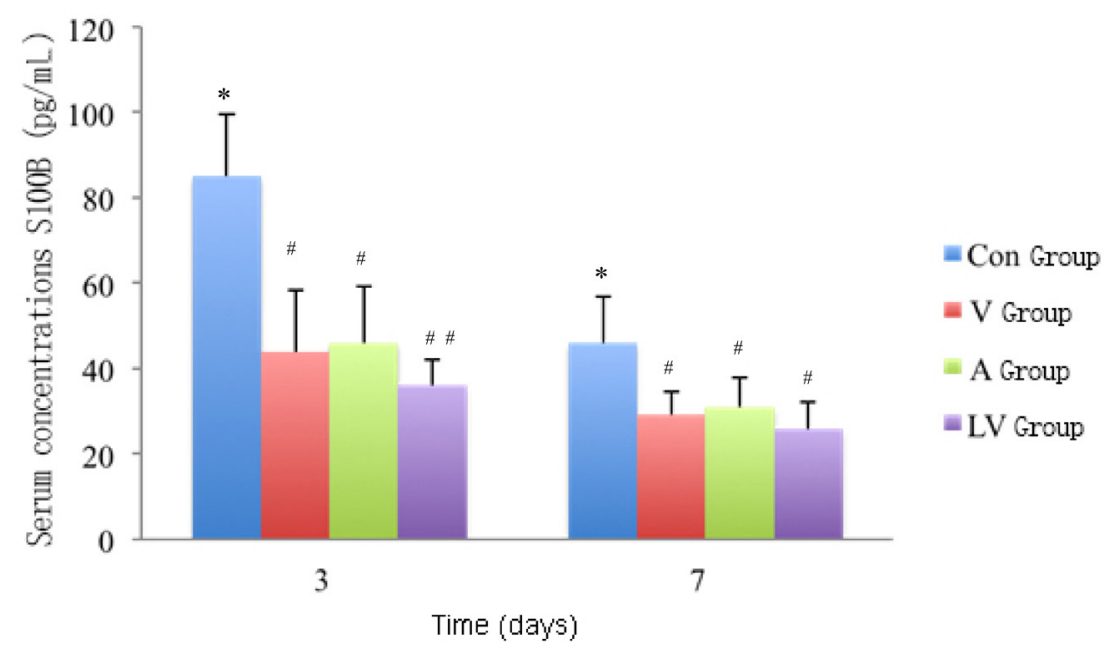

Figure 5. Detection of the $\mathrm{S} 100 \mathrm{~B}$ level in each group. ${ }^{\#} \mathrm{P}<0.01$ vs Group Con; ${ }^{*} \mathrm{P}<0.05$ vs Group Con; *P $<0.05$ vs Group LV. For group abbreviations, see legend to Figure 1. 
A

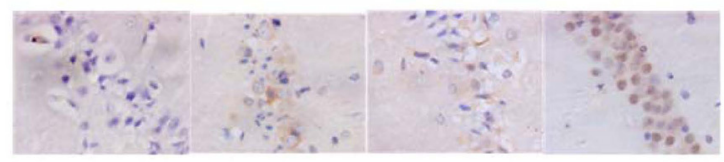

B

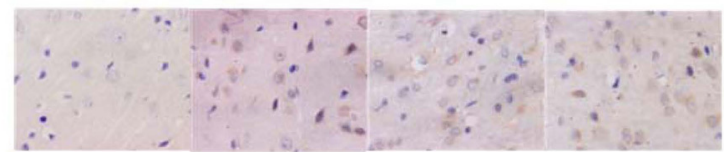

C

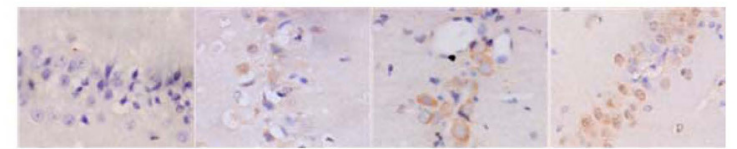

D

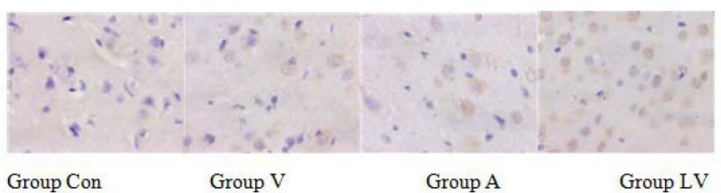

Figure 6. A. Results of VEGF by immunohistochemical method in the hippocampus in different groups. B. Results of VEGF by immunohistochemical method in the temporal cortex in different groups. C. Results of BDNF by immunohistochemical method in the hippocampus in different groups. D. Results of BDNF by immunohistochemical method in the temporal cortex in different groups. For group abbreviations, see legend to Figure 1.

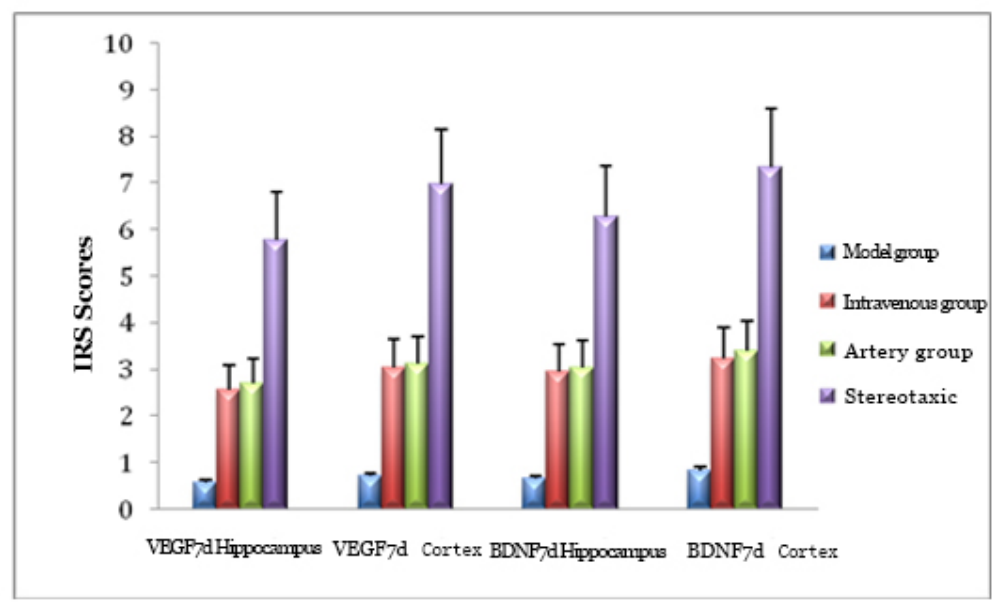

Figure 7. Comparision of immunoreactive scores (IRS) in different groups.

\section{DISCUSSION}

The results of our study suggested that BMSCs could significantly improve neurological function after cardiopulmonary resuscitation. The different migration paths used had the same therapeutic effect, but the stereotactic intracerebroventricular injection approach may be the method of transplantation. 
BMSCs are another type of stem cells in addition to hematopoietic stem cells that are present in the bone marrow. BMSCs are pluripotent and have the ability to self-replicate. In vitro studies have found that undifferentiated BMSCs could not induce an allogeneic lymphocyte proliferative response (He et al., 2013; Das et al., 2013), which suggested that BMSCs are naturally non-immunogenic, and provided the basis and prerequisite for its allograft. Some studies confirmed that transplanted BMSC treatment could improve the animal's behavioral score, learning, and memory, induce the secretion of nutritional factors and chemokines, reduce the inflammatory response, reduce the hemorrhage of the surrounding area, apoptosis, and necrosis, and increase microvascular-activated cell self-repair (Krampera et al., 2003; Wei et al., 2012; Babaei et al., 2012; He et al., 2013). BMSCs can migrate to the lesion to express neuronspecific enolase, neurofilament protein, and nestin nerve cell markers.

Bao et al. (2011) found that after cerebral infarction, BMSC transplantation could reduce the apoptosis of nerve cells, so that a large number of neurons survived in the penumbra, reduced the infarct size, and promoted recovery of neurological function. Deng and Wang (2007) extracted autologous bone marrow cells, which were isolated and cultured in vitro after a BMSC suspension transplant in the brain by intravenous infusion. After treating 5 patients with central nervous system damage, the results showed that none of the patients had adverse reactions, and symptoms of neurological deficit improved. Lee et al. (2010) conducted a 5-year follow-up study of 16 patients with middle cerebral artery territory ischemia who received transvenous BMSC transplantations. The mortality rate of the transplantation group was $25 \%$, whereas the control group mortality rate reached as high as $58.39 \%$. The transplant group and the control group showed no significant differences in terms of complications and adverse reactions (Lee et al., 2010). These experiments showed that the transplantation of bone marrow mesenchymal stem cells could effectively improve the neurological function of defects of ischemic stroke.

In this study, rats received BMSC transplants in the experimental groups after recovering for 3 and 7 days. The neurological score of the transplant groups was significantly higher than that of the control group. In addition, significant differences were found depending on the method used to line BMSCs injection. The function of stereotactic lateral ventricle injection routes was better than that by other means. Intracerebroventricular stereotactic injection is a popular way to transplant stem cells in animal experiments and clinical studies, which has the advantages of positioning accuracy, short operation time, and low surgical trauma. The stem cells can be concentrated to the lesion, and its surroundings play a therapeutic role to improve the neurological function directly and rapidly. This study showed obvious proliferation of BMCs in the hippocampus region, but there was no significant difference in the cortical regions' colonization distribution between groups treated with intravenous and intra-arterial injection routes.

In summary, BMSC treatment significantly improved neurological function after cardiopulmonary resuscitation, and stereotactic intracerebroventricular injection appears to be an ideal transplantation method.

\section{REFERENCES}

Babaei P, Tehrani BS and Alizadeh A (2012). Transplanted bone marrow mesenchymal stem cells improve memory in rat models of Alzheimer's disease. Stem Cells Int. 2012: Article ID 369417.

Bao XJ, Wei J, Feng M, Lu S, et al. (2011). Between the transplanted bone marrow mesenchymal stem cells on neuronal apoptosis after infarction rats. Chin. J. Plast. Surg. 2: 19-23. 
Das B, Kashino SS, Pulu I, Kalita D, et al. (2013). CD271(+) bone marrow mesenchymal stem cells may provide a niche for dormant Mycobacterium tuberculosis. Sci. Transl. Med. 5: 170ra13.

Deng ZF and Wang Y (2007). Ectomesenchymal thousands of autologous bone marrow cell transplantation in treatment of central nervous system injury disease (five cases report attached). Shi Yong Lin Chuang Yi Xue 70: 62-65.

$\mathrm{He} \mathrm{C}$, He C, Mo J, Fan X, et al. (2013). In vitro construction of tissue-engineered bone with bone morphogenetic protein2-transfected rabbit bone marrow mesenchymal stem cells and hydroxyapatite nanocomposite. Biomed. Tech. 58: 97-104.

Krampera M, Glennie S, Dyson J, Scott D, et al. (2003). Bone marrow mesenchymal stem cells inhibit the response of naive and memory antigen-specific T cells to their cognate peptide. Blood 101: 3722-3729.

Lee JS, Hong JM, Moon GJ, Lee PH, et al. (2010). A long-term follow-up study of intravenous autologous mesenchymal stem cell transplantation in patients with ischemic stroke. Stem Cells 28: 1099-1106.

Shi L and Yang X (2012). Differentiation potential and application of stem cells from adipose tissue. Zhongguo Xiu. Fu Chong. Jian. Wai Ke. Za Zhi. 26: 1007-1011.

Wang Y, Xu F, Zhang C, Lei D, et al. (2011). High MR sensitive fluorescent magnetite nanocluster for stem cell tracking in ischemic mouse brain. Nanomedicine 7: 1009-1019.

Wei L, Fraser JL, Lu ZY, Hu X, et al. (2012). Transplantation of hypoxia preconditioned bone marrow mesenchymal stem cells enhances angiogenesis and neurogenesis after cerebral ischemia in rats. Neurobiol. Dis. 46: 635-645.

Wen Z, Zheng S, Zhou C, Yuan W, et al. (2012). Bone marrow mesenchymal stem cells for post-myocardial infarction cardiac repair: microRNAs as novel regulators. J. Cell Mol. Med. 16: 657-671.

Williams AR, Hatzistergos KE, Addicott B, McCall F, et al. (2013). Enhanced effect of combining human cardiac stem cells and bone marrow mesenchymal stem cells to reduce infarct size and to restore cardiac function after myocardial infarction. Circulation 127: 213-223. 